\title{
RESTAURACION AMBIENTAL, SOCIAL Y TERRITORIAL FRENTE A LOS IMPACTOS GENERADOS POR LOS EMBALSES
}

\author{
Alfredo OLLERO OJEDA \\ Departamento de Geografía, Prehistoria y Arqueología \\ Universidad del País Vasco
}

Resumen: Los embalses son obras públicas altamente modificadoras del medio físico y humano. Los impactos son especialmente negativos en su entorno directo $y$ en el pasillo fluvial aguas abajo. En el presente trabajo planteamos medidas de restauración ambiental y socio-cultural de las zonas afectadas, con el objetivo de integrar estos "lagos artificiales" en el territorio, que su afección al paisaje y al medio ecológico sea mínima y que sean capaces de crear beneficios también en la propia comarca donde se construyen.

Palabras clave: Embalse, impacto, restauración ambiental, restitución territorial, uso recreativo, ecoturismo, corredor fluvial.

\begin{abstract}
Reservoirs are public works that highly modify the environment and human landscape. Impacts are specially harmful for their own environment and the riparian corridor downstream. In this work we propose some environmental and socio-cultural steps in the damaged areas in order to integrate these "artificial lakes" in the territory, to try that their influence on the landscape and the ecological environment be the smallest, and to make them able to give profits also to the region where they are built.
\end{abstract}

Key words: Reservoir, impact, environmental restoration, recreational use, ecotourism, riparian corridor. 


\section{INTRODUCCION}

Los embalses producen notables modificaciones en los sistemas fluviales, tanto hidrológicas como geomorfológicas, biológicas, paisajísticas y sociales, surgiendo un sistema distinto que precisa una gestión integral.

Esta gestión integral del sistema fluvial no debe limitarse a la explotación del recurso hídrico, objetivo meramente productivo casi exclusivo de la Política Hidráulica del Estado hasta hace pocas fechas. Debe considerar el territorio circundante al embalse, las comunidades humanas que en él habitan, la calidad del agua, de los ecosistemas y del paisaje y otros aprovechamientos alternativos del dominio público.

Precisamente la limitación a los fines productivos del recurso ha desencadenado importantes contradicciones con los usos del territorio, en aspectos ecológicos, paisajísticos y socioeconómicos, con el consiguiente proceso de convulsión social. Los embalses proyectados siguen siendo objeto de repulsa de los afectados y de los grupos que defienden el patrimonio natural y cultural. En efecto, son obras enormemente impactantes, y en algunos casos se puede discutir su utilidad. Pero en este trabajo no vamos a entrar en esa polémica sino en la búsqueda de soluciones para minimizar impactos y lograr aprovechamientos alternativos que conviertan al embalse en un elemento lo más inocuo posible para el medio natural y lo más beneficioso para su entorno humano directo, de manera que pueda compensar, en cierta medida, los perjuicios generados por su propia construcción.

En los últimos años ha tenido lugar un incremento de actividades de tipo recreativo en los embalses ya existentes, actividades que han surgido en la mayor parte de los casos de forma espontánea, no planificada ni regulada.

Ante la creciente demanda social de espacios para ocio y alternativas de desarrollo para el medio rural, los embalses son una buena solución, pero las nuevas actividades deben estar bien planificadas para que sean compatibles con el entorno ambiental y con los usos del agua.

Los embalses realizados en el pasado han implicado despoblación y desequilibrios territoriales. Los nuevos embalses y su restauración ambiental deben implicar la fijación de la población del entorno mediante el fomento del turismo y la creación de puestos de trabajo en sus proximidades. Es imprescindible socialmente beneficiar a estas zonas que aportan el agua que luego enriquece a los que aguas abajo la emplean. 


\section{IMPACTOS DE LOS EMBALSES EN EL MEDIO NATURAL}

Derivan tanto de la infraestructura en sí como de su propio proceso de construcción.

Los trabajos de construcción de la presa generan contaminación de la corriente fluvial, incremento de caudal sólido y turbidez (derivación de aguas durante la construcción y descargas bruscas, movimientos de tierras, etc.), contaminación del aire, ruidos y vibraciones. Requieren talas de vegetación, desmontes y apertura de canteras, que causan graves impactos en el paisaje y en la geomorfología local. Se originan depósitos de residuos sólidos y el transporte de materiales genera polvo y ruido.

La presa origina también un notable impacto paisajístico por desaparición de elementos naturales, introducción de elementos extraños, modificaciones cromáticas, variación y limitación de amplitudes panorámicas, etc. A la propia presa hay que añadir las edificaciones anejas y otros elementos, como las nuevas líneas de electricidad y teléfono. Los nuevos tramos de carretera, caminos y ramales necesarios, con sus correspondientes terraplenes, generan igualmente impactos en el paisaje y en la geomorfología, pudiendo provocar además problemas concretos de erosión.

La inundación supone una pérdida irreparable del paisaje primitivo y una drástica alteración de las condiciones naturales de cauce y riberas: anchura, profundidad, composición del lecho rocoso y de las orillas, caudal y régimen, temperatura, composición química de las aguas, eutrofización, modificación de las comunidades animales y vegetales del propio río y sus riberas e impacto en los usos del suelo en las orillas.

El embalsamiento de agua causa alteraciones sobre el nivel freático y en los acuíferos. También se producen variaciones en el microclima local, con incrementos de la evaporación, de las nieblas y de la humedad ambiental, siempre en función de la extensión del lago artificial y de factores como altitud, orientación del valle, etc.

El embalse altera notablemente el régimen, las características y la calidad de las aguas circulantes aguas abajo de la presa. El caudal y el régimen, dirigidos ahora por las demandas, pueden ser totalmente diferentes a los naturales, disminuyendo también las puntas de avenidas. Se producen importantes retenciones de caudal sólido, y aguas abajo de la presa se incrementa la erosión lineal en el cauce menor y desciende el nivel freático. Todo ello afecta considerablemente a los ecosistemas ribereños, y en este sentido es fundamental la garantía de unos caudales minimos.

El embalse alterará la calidad de las aguas circulantes y sus condiciones térmicas, registrándose en verano en muchos de los embalses actuales diferencias superiores a $\operatorname{los} 10^{\circ} \mathrm{C}$ entre las frías aguas que salen del embalse y las del río. 
El funcionamiento del embalse como ecosistema y la composición y grado trófico del agua embalsada están condicionados por el clima y las características físicas y humanas de la cuenca vertiente. Un buen estado ecológico de la cuenca redundará en la calidad del agua embalsada.

El tiempo de residencia elevado del agua dentro de los embalses, en contraposición a los ríos, permite el desarrollo de comunidades fitoplanctónicas reguladas por las gradaciones térmicas entre las capas superficiales y el hipolimnion a lo largo de las estaciones. Es el aporte continuado de nutrientes procedentes de las aguas residuales o de los fertilizantes agrarios el que genera una tensión sobre el ecosistema acuático que deriva en un proceso de eutrofización. El aporte de fósforo es la causa primaria de la eutrofización, pero también inciden otros nutrientes -nitrógeno, sílice-, y factores como la turbiedad del agua y la profundidad, que condicionan la luminosidad y la reserva de oxígeno disponible.

Hay dos procesos de eutrofización, uno natural (lento e imperceptible aporte de nutrientes acompañado de aterramiento por la erosión de la cuenca) y otro cultural, más grave y rápido, resultado de usos humanos en la cuenca vertiente: aguas residuales domésticas, aguas de escorrentía de terrenos agrícolas y forestales, aguas residuales industriales, aguas de lluvia que arrastran compuestos nitrogenados y fosfatados, partículas provenientes de otras cuencas arrastradas por el viento... La llegada de estos nutrientes al embalse origina aumento de biomasa de los organismos fotosintetizadores (fitoplancton autótrofo y plantas acuáticas en general) cuya incidencia se transmite a lo largo de toda la cadena trófica.

El embalse supone una barrera ecológica que fragmenta el río e impide el desarrollo normal de sus flujos de vida, rompiendo el transporte de elementos inertes (agua y minerales) y vivos (microorganismos, animales y plantas). Con ello, el río pierde su función de corredor ecológico. Además, el cambio geoecológico y medioambiental de río a lago provoca una importante pérdida de la diversidad de especies.

Las oscilaciones del agua embalsada generan una banda de desierto ecológico, sin asiento vegetal y sin hábitats, que puede alcanzar en cola amplias superficies y resulta muy impactante visualmente en períodos de aguas bajas. En esta franja, los conos de deyección y los escarpes de terraza al quedar inundados y sin vegetación pueden deshacerse con el oleaje. Las alternativas de humectación y secado pueden originar desplomes y deslizamientos en la orilla que pueden contribuir al relleno del vaso. Estos desplomes originan pequeños descensos del nivel de base de los torrentes que pueden inducir a los barrancos a incrementar su erosión lineal y remontante. 
El despoblamiento y el abandono de actividades agropecuarias derivados del embalse tienen también consecuencias sobre el medio natural al suponer una profunda modificación del conjunto de factores que durante siglos lo modelaron.

\section{IMPACTOS SOCIOECONOMICOS Y CULTURALES}

Los embalses producen beneficios, impactos positivos, en las zonas que aprovecharán el agua regulada, pero suponen la inundación de tramos de valle cuyos usos humanos del territorio deben ser abandonados. En las dos últimas décadas, después de episodios socialmente lamentables en la mente de todos, se buscan las alternativas menos impactantes socialmente para ubicar los embalses. La construcción de obras hidráulicas ha asistido a una significativa desaceleración, en buena medida por las fuertes oposiciones sociales con que la mayor parte de los proyectos han contado.

Los impactos sociales, económicos y culturales son menos generalizables que los producidos en el medio natural. Hay embalses que sólo inundan terrenos improductivos, mientras otros ocupan ricas vegas, núcleos de población, vías de comunicación y elementos del patrimonio cultural. Si hay población próxima al embalse pero no afectada por la expropiación, sufrirá molestias por la construcción de la presa: ruido, presencia de polvo y humo, tránsito de camiones y maquinaria, etc.

En general, el embalse causará pérdida de terrenos con uso agropecuario, industrial o urbano, abastecimientos de agua, vías de comunicación y todo tipo de infraestructuras. Pero por encima de pérdidas concretas, cuantificables, surgen también las rupturas de relaciones socioeconómicas entre comunidades, la desvertebración de comarcas enteras, las sensaciones de miedo ante la posible rotura de la presa, de desarraigo ante el cambio obligado de domicilio y de trabajo, de impotencia, falta de libertad y desinformación ante la decisión del embalse en las altas instancias planificadoras, de injusticia en definitiva, porque las compensaciones económicas pueden suplir un campo o una casa, pero no las vivencias y los recuerdos de cada habitante obligado a partir.

El embalse comienza a impactar en la población muchos años antes de su construcción, a lo largo de toda su fase de ideas, estudios de viabilidad y proyectos, filtrados a los afectados a través de rumores. El conocimiento de la posibilidad del embalse, la incertidumbre, se conoce sociológicamente como "sombra de percepción de riesgo", y ha sido condicionante de las actividades de la comunidad afectada, que en muchos casos ha bloqueado el interés por rentabilizar sus actividades o por realizar mejoras o inversiones de cualquier tipo. Se llega a detener el desarrollo económico del 
territorio afectado y a favorecer la fuga de los capitales familiares ahorrados. Los jóvenes que dudaban en abandonar el pueblo se deciden a hacerlo ante la posibilidad del embalse.

Por otra parte, la falta de información y el hecho de que los decisores del embalse no contaran con los habitantes afectados ha causado sentimientos de opresión, agravios y agresividad. La certeza de que no se cuenta con ellos, se les desprecia, infravalora o ignora, desemboca en una conciencia de "avasallamiento" (C.H.E., 1993).

Esta puede motivar una reacción que resulta positiva para los impulsos de reconversión y modernización de la estructura económica tradicional. Los afectados se organizan y son los jóvenes que deciden quedarse en el pueblo los que encabezan la oposición al embalse. El pueblo se esfuerza entonces por demostrar su vitalidad, se abordan proyectos de mejora y surgen iniciativas culturales, para mostrar los enormes valores que con ese embalse se perderían. Se desarrollan entonces las solidaridades locales y comarcales. De la resignación se ha pasado a la lucha, actuándose con notable unidad, con sentimientos crecientes de soberanía local. Pero aparece también el conflicto territorial entre afectados y beneficiados, entre la montaña y el llano, que puede llenar las páginas de los periódicos y politizarse.

\section{ACTUACIONES CORRECTORAS}

Frente a los impactos originados por los embalses, planteamos a continuación una serie de medidas correctoras. Unas se encaminan específicamente a corregir los impactos sobre el medio físico, otras las afecciones sobre el medio humano, y un tercer grupo engloba actuaciones integradas de carácter territorial, recreativo y ambiental que tratan de solucionar de forma conjunta los problemas ambientales y sociales del entorno del embalse.

\section{Restauración ambiental}

a) Control ambiental de las obras

Los trabajos de restauración ambiental y paisajística de las obras realizadas deben iniciarse al mismo tiempo que la construcción y proseguirse posteriormente. Los propios sistemas constructivos tienen que ser todo lo respetuosos que sea posible con el medio, limitando la ocupación de terrenos anejos para instalaciones provisionales. Los Estudios de Impacto Ambiental de las presas proponen las sugerencias y medidas correctoras durante la fase de obra que exponemos a continuación: 
-Es recomendable la instalación de pantallas acústicas y visuales (hileras tupidas de árboles) en los lugares (carretera, pueblos...) desde donde la presa y sus infraestructuras anejas sean visibles. Se conservarán con la obra concluida.

-Las canteras para extracción de materiales debrían instalarse preferentemente dentro del vaso a inundar, y también serán apantalladas o camufladas en la medida de lo posible.

-Es preciso limitar la emisión de polvo, para lo cual se propone evitar las extracciones y lugares de acopio en puntos con viento, cubrirlos o rociarlos de agua y regará con coches-cuba las rutas de transporte.

-Es fundamental durante la construcción de la presa el filtrado o decantación de las aguas efluentes, mantener un caudal mínimo pese al desvío de caudales y depurar las aguas residuales de oficinas y obras anejas a la construcción.

-Si es preciso realizar talas hay que respetar en la medida de lo posible los ejemplares arbóreos singulares o sobresalientes por su tamaño o edad, o en todo caso trasplantarlos.

b) Restauración de las obras y áreas degradadas adyacentes

Una vez finalizada la presa y los nuevos tramos de carretera, caminos y ramales debe procederse a restaurar todas las superficies alteradas, eliminando residuos y escombros. También es fundamental la descompactación de los suelos afectados por maquinaria, la eliminación y revegetación de pistas usadas para la obra, la restauración de las canteras, la sujeción de taludes de caminos y carreteras, recubriéndolos con tierra vegetal procedente del propio vaso del embalse, para luego sembrar e introducir plantaciones con especies autóctonas para integrarlos en el paisaje.

c) Corrección paisajística de la presa

Una vez construida, deben mantenerse las pantallas visuales vegetales que la camuflen en la medida de lo posible. Si es una presa de materiales sueltos puede vegetarse su paramento externo con suelo y césped.

d) Caudales mínimos

En los Estudios de Impacto Ambiental se propone como caudal mínimo cualitativo o caudal ecológico, aquél que garantizaría la conservación de la vida, el 
movimiento y la reproducción de las especies, el definido bien por la legislación francesa (el 10\% del caudal medio anual), bien por la suiza (el caudal superado en 347 días al año). Sin embargo, no podemos limitarnos a criterios estadísticos. Es preciso un profundo estudio analizando los valores de caudales medios y estiajes y la necesaria dilución los vertidos aguas abajo de la presa. El caudal mínimo debe ser acorde con la época del año y los ciclos vitales de las especies, es decir, deberíamos hablar de régimen ecológico, y suficiente para mantener una lámina de agua corriente continua.

Paralelamente a descargar un caudal suficiente para el mantenimiento de los ecosistemas aguas abajo, es fundamental que el agua vertida proceda de diferentes profundidades del embalse (torre de tomas), para controlar la temperatura y la calidad.

e) Medidas contra la eutrofización

La más importante es la depuración de los vertidos directos al río aguas arriba del embalse. Si el uso del embalse es para agua de boca, la depuración total es imprescindible. La garantía de contar con agua embalsada de buena calidad será también un valor ecológico y un atractivo para el turismo. Las aguas residuales urbanas de localidades pequeñas y las derivadas de industrias agroalimentarias pueden depurarse mediante filtros verdes, aprovechándolas para el riego de explotaciones forestales (choperas). Las aguas residuales de los núcleos grandes deben ser sometidas a tratamiento de depuración físico-química, con precipitación de fósforo y stripping de nitrógeno. También es posible transportar, embalsar y reutilizar el agua resultante para regar, crear balsas discontinuas rodeadas de plantaciones forestales, hacer un parque con tratamiento paisajístico o incluso un campo de golf.

Además de depurar los vertidos controlados es necesario eliminar los basureros y escombreras incontrolados que proliferan en las riberas.

Es fundamental para evitar una rápida eutrofización el deforestar previamente el vaso del embalse, eliminando toda la tierra vegetal, útil para plantaciones y restauraciones, y toda la vegetación, trasplantando a la orilla los ejemplares interesantes. Todo ello contribuye además al uso recreativo, ya que se eliminan obstáculos para el baño o el uso náutico.

Es útil la conservación de las masas forestales de cabecera, de los matorrales próximos al vaso y de la vegetación ribereña para evitar pérdidas de suelo con el consiguiente aporte difuso de nutrientes.

Otra medida poco empleada es la de diversificar los cauces de entrada de los ríos y barrancos afluentes, excavando pequeños canales en el terreno para incrementar 
la superficie de intercambio entre el agua y los órganos de captación de nutrientes de las plantas.

También es posible incrementar la tasa de renovación hidráulica del epilimnion, mediante suelta selectiva a ese nivel, para mantener una baja relación epilimnion/hipolimnion que permita a este último asimilar los materiales oxidables desde las capas superficiales.

Es muy recomendable instalar una estación de control de calidad y tratamiento del agua, con labores de restitución de oxígeno, floculación de fósforo, eliminación de algas, etc. Se puede repoblar el embalse con especies piscícolas plantófagas, como los géneros Coregonus y Alburnus, que eliminan materia orgánica del vaso.

f) Restauración de riberas y del corredor ecológico fluvial

Tras la construcción del embalse es preciso realizar una amplia serie de labores de restauración de riberas tanto en el sector inmediato aguas arriba y de cola como en las orillas del nuevo lago, así como aguas abajo de la presa.

Deberían reponerse las pérdidas vegetales, recuperar en la orilla la población de macro-invertebrados bénticos y crear hábitats y frezaderos similares a los destruidos, siendo útiles los tramos finales de barrancos afluentes. También pueden acondicionarse bebederos para animales terrestres. Es factible la repoblación piscícola con especies autóctonas, buscando también el atractivo de la pesca, que puede redundar en beneficios socioeconómicos.

Las presas son barreras infranqueables para la ictiofauna. Es preciso crear sistemas para favorecer el desplazamiento de peces a través de la presa, fundamentalmente escalas o canales.

Atenuar el desierto ecológico por oscilación de las orillas del embalse es una labor sumamente difícil. Se pueden revegetar las orillas con carrizos y aneas para reducir los procesos de solifluxión y deslizamientos en la banda de fluctuación del agua. Lo más útil es levantar pequeñas presas de cola a la cota de máximo embalse normal para mantener el nivel y facilitar la pervivencia de pequeños ecosistemas de ribera.

Aguas abajo de la presa hay que evitar la erosión lineal y el descenso del nivel freático tratando de mantener la lámina de agua del cauce elevada, bien con pequeños azudes sucesivos, bien con un caudal siempre suficiente. 
g) Mejora forestal en la cuenca vertiente

Se trata de una medida correctora indirecta que beneficia al embalse, reduciendo la velocidad de aterramiento, así como a todo el sistema natural en que se integra. La reforestación contribuye también a compensar las superficies vegetales perdidas por el embalsamiento. Las labores de repoblación y mejora pueden aplicarse por toda la cuenca vertiente y en las laderas directas del vaso del embalse.

\section{Medidas compensatorias para el medio socioeconómico}

a) Información y participación en la toma de decisiones

Es imprescindible reconocer como parte a los afectados y fundamental el diálogo continuo en la toma de decisiones, en las condiciones de regulación y en la negociación de las compensaciones. La información de los afectados debe ser total desde el primer momento, con canales de comunicación fluidos y estables, para evitar malentendidos y sensaciones negativas relacionadas con la sombra de percepción de riesgo. Es imprescindible una mayor transparencia en relación a las necesidades reales y a las intenciones últimas que inspiran los proyectos (C.H.E., 1993).

b) Indemnizaciones

Deben pagarse con celeridad indemnizaciones justas que compensen tanto los elementos cuantificables como los cualitativos, relacionados con la afección que la obra produce para la subsistencia y futuro de un individuo, familia, comunidad o comarca.

Ahora bien, un tratamiento exclusivamente pecuniario no contribuye necesariamente a restaurar el territorio. La política compensatoria debe ser mucho más amplia que la mera indemnización.

c) Reposiciones

Es preciso reponer con celeridad, ya durante las obras del embalse, las vías de comunicación y los servicios afectados (líneas eléctricas y telefónicas, conducciones de agua, etc.), así como trasladar los elementos de valor cultural que quedarían anegados.

d) Riegos de compensación

Al margen de los aprovechamientos de las aguas del embalse aguas abajo, habría que crear riegos de compensación para los pueblos afectados, sobre superficies al menos iguales a las perdidas como consecuencia de la expropiación derivada del 
embalse. A la par, habría que dotar a la zona de mejoras como concentración parcelaria, sistemas de riegos a la demanda y por goteo, subvenciones para nuevas inversiones, etc.

\section{e) Fomento de empleo}

Hay que compensar las posibles pérdidas de trabajo causadas por el embalse y fomentar el empleo en toda la comarca afectada, favoreciendo la inversión y tratando de que los nuevos puestos de trabajo relacionados con el embalse sean ocupados por personas de la comarca. También puede tener un efecto positivo el aprovechamiento de la nueva presa para la instalación de una minicentral gestionada por el municipio o mancomunidad afectados. La potenciación de actividades recreativas relacionadas con el embalse también puede generar varios puestos de trabajo, así como el fomento de los valores paisajísticos, históricos y culturales de la comarca, que animen a inversiones en turismo rural, tal como expondremos más adelante. Igualmente se crearía empleo ejecutando los trabajos planteados de restauración y mejora ambiental.

\section{Actuaciones globales de ordenación y protección}

a) Pacto territorial

Hay que evitar los conflictos territoriales compensando a las comarcas afectadas de forma global y en un sentido territorial lo más amplio posible. El contexto en que deben ubicarse las demandas de los afectados es el del esfuerzo de reconversión y modernización económica de la comarca ante la crisis del modelo tradicional, promoviendo la mejora y rentabilidad de las explotaciones agropecuarias, la mejora en las comunicaciones y servicios sociales y la inversión en turismo, como medidas más útiles en la situación actual de las comarcas rurales deprimidas. Los afectados pueden así pasar de criticar las intervenciones impositivas a reclamar beneficios, dejar de considerarse víctimas y pasar a tomar conciencia de su soberanía territorial y de su derecho a ser tan beneficiados como las comarcas que van a aprovechar el agua embalsada (C.H.E., 1993).

Por ello, es fundamental formalizar un pacto territorial que favorezca la compatibilidad de intereses entre las zonas de servidumbre y las beneficiarias, y conciba integralmente las necesidades del territorio afectado. Así, la obra pública no puede circunscribirse exclusivamente a la planificación hidrológica, sino integrarse totalmente en la planificación territorial. La obra nunca puede ser entendida sóla, sino inseparable de sus consecuencias y de las medidas de compensación. El embalse debería ser acompañado de un plan integral de restitución territorial y desarrollo sostenido del valle afectado, y de planes locales de mejora de servicios y dotaciones. 


\section{b) Uso recreativo y sensibilización ambiental}

Los embalses pueden tener un papel ambiental-social de primer orden. Su carácter de paisaje nuevo, atractivo por la presencia de agua, le confiere la posibilidad de ejercer de área de esparcimiento disuasoria que atraiga y concentre a visitantes que, en busca de un disfrute ambiental y recreativo, acudirían a enclaves próximos de mayor valor natural y fragilidad ecológica. A la función ecológica por el citado papel disuasorio que el embalse puede ejercer cabe añadir una importante función social, tanto para los visitantes potenciales, que pueden contar con un nuevo punto de esparcimiento, como para la comarca rural afectada, que se verá beneficiada por ingresos extra provenientes del nuevo atractivo turístico que puede constituir el embalse y su entorno directo.

Paralelamente al uso recreativo hay que lograr la sensibilización ambiental de los usuarios, desde la mera interpretación del paisaje hasta el conocimiento de la situación y problemática comarcal y del papel del embalse en su contexto territorial. Pueden desarrollarse las siguientes acciones:

- Areas recreativas equipadas en las inmediaciones del embalse que incluyan elementos informativos. Preferentemente se ubicarían en lugares bien comunicados, con buenas panorámicas sobre el embalse (mirador) y necesitados de restauración (por ejemplo, las propias canteras).

- Jardín botánico de especies autóctonas, que incluya ejemplares singulares rescatados del terreno embalsado.

- Puede destinarse terreno de las laderas directas al embalse para la ubicación de segundas residencias, siguiendo normas constructivas compatibles con el paisaje y la arquitectura popular comarcal.

- Embarcadero y centro de actividades acuáticas.

- Playas artificiales y zonas de baño y pesca señalizadas.

- Teniendo en cuenta que las oscilaciones de nivel de muchos embalses son muy fuertes, y que en verano, época de máxima demanda de ocio, pueden presentar estiajes profundos que impidan las prácticas recreativas, en dichos casos sería útil la construcción de pequeños embalses de cola para uso recreativo de baja densidad (baño y pesca), que puede ser compatible con el mantenimiento de un valioso ecosistema palustre. 
- Senderos educativos y deportivos por todo el entorno del embalse.

- Centros de interpretación, aulas de naturaleza, albergues educativos, zonas de acampada. Pueden emplearse edificios valiosos afectados por el embalse y trasladados.

- Establecimiento de un Corredor Verde Fluvial para el conjunto del río afectado, aguas arriba y abajo del embalse y en las riberas de éste. Tendría funciones ecológica de conexión de ecosistemas, protectora de las riberas del río, del nuevo embalse y del entorno paisajístico, y ecoturística al promover, concentrar y controlar una serie de actividades poco impactantes y que pueden beneficiar desde el punto de vista socio-económico-cultural a los habitantes del valle: espacios recreativos, posibilidad de practicar deportes, senderos continuos a lo largo de la ribera, alquileres de bicicletas o caballos, viviendas de turismo rural, hostelería, embellecimiento de cascos urbanos, ecomuseos, museos etnográficos, albergues, fomento de trabajos tradicionales y manifestaciones culturales, restauración de monumentos, huertas tradicionales con agricultura biológica, productos típicos, etc. En suma, el río debe ser un pasillo natural continuo para el desplazamiento de la flora y de la fauna silvestres, así como para el desplazamiento naturalístico-didáctico-cultural-recreativo-ecoturístico del hombre apreciando esos valores naturales. Generalmente es necesario fortalecer el corredor con plantaciones para densificar y extender el pasillo ribereño sobre terrenos del dominio público hidráulico.

- Promoción turística de todos los elementos reseñados.

\section{c) Protección}

Entre las implicaciones territoriales que debe conllevar la construcción del embalse se encuentra la protección legal de los espacios naturales de interés de la cuenca vertiente.

Deben deslindarse las riberas en todo el corredor fluvial, incluyendo las del embalse, elaborar un Plan de Estimación y zonificar sus usos. Lo ideal sería crear una figura de protección de garantice el mantenimiento y la mejora ambiental de todo el tramo ribereño considerado, para que pueda conservar su continuidad como corredor ecológico, reglamentando los usos recreativos y los espacios más aptos para los mismos. 
Paralelamente se puede solucionar la problemática local por extracciones de áridos, invasiones del dominio público hidráulico, etc. Los trabajos de defensa existentes en el tramo de río a restaurar pueden ser objeto de un tratamiento paisajístico mediante cortinas verdes arbóreas y arbustivas.

\section{CONCLUSIONES}

Es preciso concebir los embalses, desde el mismo momento en que son planteados como posibilidad, como elementos integrados en un complejo contexto territorial y participantes en la ordenación territorial de comarcas extensas, sobre las que sus impactos ambientales y sociales son considerables.

La integración de los embalses en la ordenación del territorio implica llevar a cabo medidas correctoras en tres frentes:

a) específicas contra los impactos ambientales,

b) específicas contra los impactos socioeconómicos y culturales, y

c) globales o integradas de restitución territorial

Las medidas del tercer frente no tienen por qué ser las más costosas económicamente, pero sí son las más complejas, y requieren un pacto territorial de la Administración con los afectados y beneficiados. En la situación actual del medio rural y contando con la alta demanda de ocio de las poblaciones urbanas, los usos recreativos derivados del embalse y la creación de corredores verdes de desarrollo ecoturístico siguiendo el valle afectado, pueden ser los instrumentos más útiles para la creación de riqueza y empleo en las comunidades afectadas. Pero deben ser acompañados de mejoras en explotaciones agropecuarias, servicios y comunicaciones, así como de la protección del medio natural en las cuencas vertientes a los embalses, imprescindible para conservar ecosistemas y una calidad del agua y del paisaje que son las bases para la atracción ecoturística.

\section{BIBLIOGRAFÍA}

AVILES GARCIA, J. (1994): Control de la eutrofización. Curso sobre eutrofización de embalses $e$ indicadores biológicos de la calidad de las aguas en los ríos, 275-281, CEDEX-MOPTMA, Madrid. 
CONFEDERACION HIDROGRAFICA DEL EBRO (1993): Estudio del impacto socio-cultural derivado de la regulación del río Esera y propuestas para un pacto territorial. Universidad de Zaragoza y Oficina de Planificación de la C.H.E.

MOPU (1989): Guías metodológicas para la elaboración de estudios de impacto ambiental. 2. Grandes presas. Monografías de la Dirección General de Medio Ambiente.

MOPTMA (1994): Programa de fomento social y adecuación ambiental de embalses. Dirección General de Obras Hidráulicas (informe sin publicar).

OLLERO OJEDA, A. (1994): Corredores fluviales y embalses en la ordenación del territorio pirenaico aragonés. Primeros Encuentros Pirenaicos para el Medio Ambiente y el Desarrollo. Comunidad de Trabajo de los Pirineos, Andorra.

ORTIZ CASAS, J.L. (1994): Indicadores de eutrofización. Criterios de clasificación de lagos y embalses según el grado de eutrofia.Curso sobre eutrofización de embalses $e$ indicadores biológicos de la calidad de las aguas en los ríos, 87-113, CEDEX-MOPTMA, Madrid.

RIERA, J.L. y MORGUI, J.A. (1990): Limnología regional de los embalses españoles. Mundo científico, $104(10), 720-726$. 